\title{
DISCRETE FREE PRODUCTS OF TWO COMPLEX CYCLIC MATRIX GROUPS
}

\author{
by RONALD J. EVANS
}

(Received 12 September, 1977)

1. Introduction. All 2-by-2 matrices in this paper are to be viewed as linear fractional transformations on the extended complex plane $\mathbb{C}^{*}$. Let $L^{+}$and $L^{-}$be the open half-planes to the right and left, respectively, of the extended imaginary axis $L$. Let $\Lambda$ be the set of complex 2-by-2 matrices $A$ with real trace and determinant \pm 1 such that $A\left(L^{+}\right) \subset L^{-}$. Let $\Omega=\Omega_{1} \cup \Omega_{2} \cup \Omega_{3} \cup \Omega_{4}$, where

$$
\begin{aligned}
& \Omega_{1}=\{A \in \Lambda: \operatorname{det} A=1,|\operatorname{tr} A| \geq 2\}, \\
& \Omega_{2}=\{A \in \Lambda: \operatorname{det} A=1,|\operatorname{tr} A|=2 \cos (\pi / q) \text { for some integer } q>2\}, \\
& \Omega_{3}=\{A \in \Lambda: \operatorname{tr} A=0\},
\end{aligned}
$$

and

$$
\Omega_{4}=\left\{A \in \Lambda: \operatorname{det} A=-1, A^{2} \in \Lambda\right\} .
$$

Observe that the elements of $\Omega_{1} \cup \Omega_{4}$ have infinite order, while those of $\Omega_{2} \cup \Omega_{3}$ have finite order.

We will prove that whenever $A \in \Omega$ and $B \in \Omega$ do not both have a fixed point on $L$, then the group $\left\langle A, B^{t}\right\rangle$ is the discrete free product $\langle A\rangle *\left\langle B^{t}\right\rangle$, where $B^{t}$ denotes the transpose of $B$. The case where both $A$ and $B$ have a fixed point on $L$ is also discussed. We show in addition that if $A \in \Omega$ and $B \in \Omega$ are real, then $\left\langle A, B^{t}\right\rangle$ is the discrete free product $\langle A\rangle *\left\langle B^{\prime}\right\rangle$ if and only if for every real $u$,

$$
\left\{A, B^{\prime}\right\} \not \subset\left\{\left|\begin{array}{rr}
1 & 0 \\
0 & -1
\end{array}\right|,\left|\begin{array}{cc}
0 & -u \\
1 / u & 0
\end{array}\right|\right\} .
$$

The significance of these results is discussed in \$3. In particular, we show there that the latter result implies that the free products in [2, Theorem 1] are all discrete. We thereby fill a gap in [2, 84$]$, wherein the discreteness was proved only in a special case.

Discrete free products of two cyclic matrix groups have been extensively studied, along different lines. For example, Purzitsky [5] has given necessary and sufficient conditions for any group $\langle A, B\rangle$ generated by real linear fractional transformations $A$ and $B$ of determinant 1 to be the discrete free product $\langle A\rangle *\langle B\rangle$. (See also [6].)

2. Definitions and notation. Let $P$ denote the set of matrices $\left|\begin{array}{ll}a & b \\ c & d\end{array}\right|$ of determinant \pm 1 such that either $a, b \geq 0 \geq c, d$ or $a, b \leq 0 \leq c, d$. We reserve the notation $M=\left|\begin{array}{ll}a & b \\ c & d\end{array}\right|$ for a matrix (or transformation) in $\Omega \cap P$. In fact, the symbol $M$ can be used to denote an arbitrary real matrix in $\Omega$, in view of Lemma 5 . Given a transformation $M$, we stipulate

Glasgow Math. J. 20 (1979) 69-80. 
without loss of generality that $a, b \geq 0 \geq c, d$. If, moreover, $c(a+d) \geq 0, M$ is said to be plussed.

Let $\omega_{M}$ and $\omega_{M}^{\prime}$ be the fixed points of $M$, with $\left|\omega_{M}\right| \leq\left|\omega_{M}^{\prime}\right|$ if the fixed points are on the extended real line $\mathbb{R}^{*}$, and with $\operatorname{Im} \omega_{M}>0$ otherwise. Note that $\omega_{M}$ is finite, because otherwise $\omega_{M}=\omega_{M}^{\prime}=\infty$ and $M$ would have the form $\left|\begin{array}{ll}1 & * \\ 0 & 1\end{array}\right|$, which contradicts the fact that $M \in P$. Note also that $\operatorname{Re} \omega_{M} \leq 0$, since $M\left(L^{+}\right) \subset L^{-}$. Let $L_{M}$ denote the extended vertical line through $\omega_{M}$. Let $L_{M}^{+}$and $L_{M}^{-}$denote the open half-planes to the right and left of $L_{M}$, respectively.

If $E$ is a nonsingular 2-by-2 matrix and $\mathscr{A}$ is a set of 2-by-2 matrices, write $\mathscr{A}^{E}=\left\{A^{E}: A \in \mathscr{A}\right\}$, where $A^{E}=E A E^{-1}$. A 2 -by-2 matrix $S$ is said to be an $L$-map if $S\left(L^{+}\right)=L^{+}$. Note that if $S$ and $S_{1}$ are $L$-maps, then so are $S^{-1}$ and $S \circ S_{1}$. In addition, $S(L)=L, S\left(L^{-}\right)=L^{-}, \Lambda^{S}=\Lambda$, and $\Omega^{S}=\Omega$.

Let $I=\left|\begin{array}{ll}1 & 0 \\ 0 & 1\end{array}\right|, T_{u}=\left|\begin{array}{cc}0 & -u \\ 1 / u & 0\end{array}\right|$, and $W_{r}=\left|\begin{array}{cc}1 & r \\ 0 & -1\end{array}\right|$. Write $T=T_{1}$ and $W=W_{0}$.

Given $U \subset \mathbb{C}^{*}$, let cl $U$ denote the closure of $U$ in $\mathbb{C}^{*}$ and let ccl $U$ denote the complement of the closure of $U$ in $\mathbb{C}^{*}$.

3. Main results. We now present the main theorems. The proofs are postponed until §6.

Theorem 1. Let $A, B \in \Omega$ and $C=B^{\mathrm{w}}$. Suppose that $A$ and $C$ do not both have a fixed point on $L$. Then $\langle A, C\rangle$ is the discrete free product $\langle A\rangle *\langle C\rangle$.

Theorem 2. Let $A, B \in \Omega$ and $C=B^{W}$. If $A$ and $B$ are real, then $\langle A, C\rangle$ is the discrete free product $\langle A\rangle *\langle C\rangle$ if and only if for every real $u,\{A, C\} \not \subset\left\{W, T_{u}\right\}$.

Consider the Hecke group $\left\langle S_{\lambda}, T\right\rangle$ generated by $S_{\lambda}=\left|\begin{array}{ll}1 & \lambda \\ 0 & 1\end{array}\right|$ and $T=\left|\begin{array}{rr}0 & -1 \\ 1 & 0\end{array}\right|$. It is well-known that when $\lambda=2 \cos (\pi / q)$ for an integer $q \geq 3$, then $\left\langle S_{\lambda}, T\right\rangle$ is the discrete free product $\left\langle T S_{\lambda}\right\rangle *\langle T\rangle$, and that when $\lambda$ is a complex number of modulus $\geq 2$, then $\left\langle S_{\lambda}, T\right\rangle$ is the discrete free product $\left\langle S_{\lambda}\right\rangle *\langle T\rangle$. Theorem 1 is sufficiently general to imply these results. For, if $\lambda=2 \cos (\pi / q)$ for an integer $q \geq 3$, apply Theorem 1 with $A=T S_{\lambda}, B=T$. If $\lambda \in \mathbb{C}$, $|\lambda| \geq 2$, apply Theorem 1 with $A=S_{\lambda}^{G}, B=T^{G}$, where $G=\left|\begin{array}{rr}-1 & 1 \\ 1 & 1\end{array}\right|$.

If, in Theorem $1, C$ is defined to be $B^{\prime}$ instead of $B^{w}$, the resulting statement is equivalent. To see this, we need only show that $\Omega^{W}=\Omega^{t}$. Assume that for some $\tau_{1} \in L^{+}$ and some $A \in \Lambda, A^{-1}\left(\tau_{1}\right)=\tau_{2} \in \operatorname{cl}\left(L^{+}\right)$. Then $A\left(\tau_{2}\right) \in L^{+}$, so for some $\tau_{3} \in L^{+}$close to $\tau_{2}$, $A\left(\tau_{3}\right) \in L^{+}$. This contradicts the fact that $A\left(L^{+}\right) \subset L^{-}$. Thus $A^{-1}\left(L^{+}\right) \subset L^{-}$for all $A \in \Lambda$.

This proves that $\Lambda=\Lambda^{-1}$. Thus $\Omega=\Omega^{-1}$; so $\Omega^{t}=T \Omega^{-1} T=\Omega^{T}$. Since $T W=\left|\begin{array}{ll}0 & 1 \\ 1 & 0\end{array}\right|$ is an $L$-map, $\Omega^{\mathrm{Tw}}=\Omega$. Therefore, $\Omega^{t}=\Omega^{\mathrm{T}}=\Omega^{\mathrm{w}}$.

Similarly, if $C$ is defined to be $B^{t}$ instead of $B^{W}$ in Theorem 2, the resulting statement is equivalent. Another equivalent formulation of Theorem 2 is as follows. "Let 
$A, B \in \Omega^{v}$, where $V=2^{-1 / 2}\left|\begin{array}{rr}1 & -1 \\ 1 & 1\end{array}\right|$, and let $C=B^{t}$. If $A$ and $B$ are real, then $\langle A, C\rangle$ is the discrete free product $\langle A\rangle *\langle C\rangle$ if and only if, for every real pair $r, s$ satisfying $s^{2}-r^{2}=1$, we have $\{A, C\} \not \subset\left\{\left|\begin{array}{cc}r & s \\ -s & -r\end{array}\right|,\left|\begin{array}{ll}0 & 1 \\ 1 & 0\end{array}\right|\right\}$ ". In the notation of [2], it follows from [2, Lemma 9] and Lemma 5 below that $J \subset \Lambda^{v}$; hence $K \subset \Omega^{v}$, so Theorem 2 now immediately implies that the free products in [2, Theorem 1] are all discrete.

Theorem 2 is, in fact, an extension of a theorem of Newman [4, Theorem 15, p. 162]. For if in Theorem 2 the determinants of $A$ and $B$ are restricted to be 1, then Theorem 2 becomes a restatement of Newman's theorem (see Lemma 5 below).

The following simple example shows that the requirement in Theorem 2 that $A$ and $B$ are real cannot be dropped. Let $A=\left|\begin{array}{cc}1 & i \\ 0 & -1\end{array}\right|$ and $C=\left|\begin{array}{rr}1 & 0 \\ i & -1\end{array}\right|$. Then $A \in \Omega, C \in \Omega^{w}$, but $\langle A, C\rangle$ is not the free product $\langle A\rangle *\langle C\rangle$, since $(A C)^{3}=I$.

4. Real conjugates of complex matrices. Theorem 4 (below) will enable us to focus attention on those matrices in $\Omega$ which are real. First a lemma is proved.

Lemma 3. If $N \in \Omega$ fixes $\infty$, then $N=W_{\rho}$ for some $\rho$. If $N \in \Omega$ fixes 0 , then $N=W_{\rho}^{t}$ for some $\rho$.

Proof. Suppose that $N(\infty)=\infty$, so $N=\left|\begin{array}{ll}\alpha & \beta \\ 0 & \delta\end{array}\right|$. For each $\tau \in L^{+}, N \tau=(\alpha / \delta) \tau+\beta / \delta \epsilon$ $L^{-}$. Let $\psi=\arg (\alpha / \delta)$ with $-\pi<\psi \leq \pi$. Suppose that $\psi \in(-\pi, 0]$. If $\tau \in L^{+}$has sufficiently large modulus and arg $\tau$ is sufficiently close to $\pi / 2$, then $N \tau \in L^{+}$, a contradiction. Suppose then that $\psi \in(0, \pi)$. If $\tau \in L^{+}$has sufficiently large modulus and $\arg \tau$ is sufficiently close to $-\pi / 2$, then $N \tau \in L^{+}$, a contradiction. Thus $\psi=\pi$, i.e., $\alpha / \delta<0$.

Suppose that $\alpha \notin \mathbb{R}$. Since $\operatorname{tr} N$ is real, $\delta=\bar{\alpha}$. Since $\alpha / \bar{\alpha}$ is a negative number of modulus $1, \alpha=-\bar{\alpha}$. Thus $N=\left|\begin{array}{rr}\alpha & \beta \\ 0 & -\alpha\end{array}\right|$, i.e., $N=W_{\rho}$ for some $\rho$.

Suppose that $\alpha \in \mathbb{R}$. Since $\alpha / \delta<0$, $\operatorname{det} N<0$. Thus $N \in \Omega_{3}$ or $N \in \Omega_{4}$. For all $\tau \in L^{+}$, $N^{2} \tau=\left(\alpha^{2} / \delta^{2}\right) \tau+\beta(\alpha+\delta) / \delta^{2}$. If $\tau \in \mathbb{R}$ is sufficiently large, then $N^{2} \tau \in L^{+}$. Thus $N^{2} \notin \Lambda$, so $N \notin \Omega_{4}$. Therefore, $N \in \Omega_{3}$. It follows that $N=\left|\begin{array}{rr}\alpha & \beta \\ 0 & -\alpha\end{array}\right|$, i.e., $N=W_{\rho}$ for some $\rho$. This proves the first assertion.

Suppose that $N \in \Omega$ fixes 0 . Then, since $T W=\left|\begin{array}{ll}0 & 1 \\ 1 & 0\end{array}\right|$ is an $L$-map, $N^{T w} \in \Omega$ and $N^{T W}$ fixes $\infty$. By the first assertion, $N^{T W}=W_{\rho}$ for some $\rho$, so $N=W_{-\rho}^{t}$.

THEOREM 4. Let $N=\left|\begin{array}{ll}\alpha & \beta \\ \gamma & \delta\end{array}\right| \in \Omega$. Then there is an $L$-map $S$ such that $S N S^{-1}$ is real.

Proof. Note that the fixed points $\omega$ and $\omega^{\prime}$ of $N$ lie in $\mathrm{cl}\left(L^{-}\right)$, since $N\left(L^{+}\right) \subset L^{-}$. Define $\omega_{1}=\operatorname{Re} \omega, \omega_{2}=\operatorname{Im} \omega, \omega_{1}^{\prime}=\operatorname{Re} \omega^{\prime}$, and $\omega_{2}^{\prime}=\operatorname{Im} \omega^{\prime}$. 
Case 1: $\omega, \omega^{\prime} \in L$. Assume that $\omega=\omega^{\prime}$. If $\omega \neq \infty$, conjugate $N$ by the $L$-map $\left|\begin{array}{rr}0 & 1 \\ 1 & -\omega\end{array}\right|$. Thus we may assume that both fixed points of $N$ are $\infty$. Then $N=\left|\begin{array}{ll}1 & \beta \\ 0 & 1\end{array}\right|$ for some $\beta$, which contradicts the fact that $N \in \Lambda$. Hence $\omega \neq \omega^{\prime}$. If neither $\omega$ nor $\omega^{\prime}$ is $\infty$, conjugate $N$ by the $L$-map $\mid \begin{array}{cc}1 & -\omega^{\prime} \\ \omega-\omega^{\prime} & -\omega\left(\omega-\omega^{\prime \prime},\left.\right|^{\prime}\right.\end{array}$; if one of $\omega$ or $\omega^{\prime}$ is $\infty$, say $\omega=\infty$, then conjugate $N$ by the $L$-map $\left|\begin{array}{cc}1 & -\omega^{\prime} \\ 0 & 1\end{array}\right|$ (which fixes $\omega=\infty$ ). Thus we may assume that $\omega^{\prime}=0$ and $\omega=\infty$. By Lemma 3, $N=W_{\rho}$ and $N=W_{\nu}^{t}$ for some pair $\rho, \nu$. Hence $N=\left|\begin{array}{rr}1 & 0 \\ 0 & -1\end{array}\right|$.

Case 2: $\omega^{\prime} \in L, \omega \in L^{-}$. If $\omega^{\prime} \neq \infty$, conjugate $N$ by the $L$-map $\left|\begin{array}{cc}0 & 1 \\ 1 & -\omega^{\prime}\end{array}\right|$. Then we may assume that $\omega^{\prime}=\infty$. We may moreover assume that $\omega=-1$, for otherwise conjugate $N$ by the $L$-map $\left|\begin{array}{cc}i & \omega_{2} \\ 0 & -i \omega_{1}\end{array}\right|$ (which fixes $\omega^{\prime}=\infty$ ). Thus $N=\left|\begin{array}{cc}\alpha & \alpha-\delta \\ 0 & \delta\end{array}\right|$. By Lemma $3, N=$ $\left|\begin{array}{rr}1 & \rho \\ 0 & -1\end{array}\right|$ for some $\rho$. Therefore, $N=\left|\begin{array}{rr}1 & 2 \\ 0 & -1\end{array}\right|$.

Case 3: $\omega, \omega^{\prime} \in L^{-}$and $\omega=\omega^{\prime}$. We may assume that $\omega=-1$, for otherwise conjugate $N$ by the $L$-map $\left|\begin{array}{cc}i & \omega_{2} \\ 0 & -i \omega_{1}\end{array}\right|$. Thus $N=\left|\begin{array}{cc}1-\gamma & -\gamma \\ \gamma & 1+\gamma\end{array}\right|$. If $\gamma \in \mathbb{R}$, then $N$ is real, so suppose that $\gamma \notin \mathbb{R}$. Let $\eta=\arg \gamma$. Let $S=\left|\begin{array}{ll}1 & k \\ k & 1\end{array}\right|$, where $k=i \tan (\eta / 2)$. Then $S$ is an $L$-map and $S N S^{-1}=\left|\begin{array}{cc}1-\gamma_{1} & -\gamma_{1} \\ \gamma_{1} & 1+\gamma_{1}\end{array}\right|$, where $\gamma_{1}=|\gamma| \in \mathbb{R}$.

Case 4: $\omega, \omega^{\prime} \in L^{-}, \omega \neq \omega^{\prime}$. We may assume that $\omega^{\prime}=-1$, otherwise conjugate $N$ by the $L$-map $\left|\begin{array}{cc}i & \omega_{2}^{\prime} \\ 0 & -i \omega_{1}^{\prime}\end{array}\right|$. Assume that $\omega_{2} \neq 0$. Let $S^{\prime}=\left|\begin{array}{cc}x i & 1 \\ 1 & x i\end{array}\right|$, where $x$ is a solution of the equation

$$
\omega_{2} x^{2}+x\left(|\omega|^{2}-1\right)-\omega_{2}=0 .
$$

Since the discriminant of this quadratic equation is

$$
\left(\omega_{1}^{2}-1\right)^{2}+\omega_{2}^{4}+2 \omega_{1}^{2} \omega_{2}^{2}+2 \omega_{2}^{2},
$$

it follows that $x \in \mathbb{R}$. Therefore $S^{\prime}$ is an $L$-map. Since $S^{\prime}(-1)=-1$ and $S^{\prime}(\omega) \in \mathbb{R}$ by definition of $x$, we see that $S^{\prime} N\left(S^{\prime}\right)^{-1}$ fixes the two distinct real points -1 and $S^{\prime}(\omega)$. It may thus be assumed without loss of generality that $\omega_{2}=0$, i.e., that $N$ fixes -1 and $\omega$, where $\omega<0, \omega \neq-1$. Write $N=\left|\begin{array}{cc}\sigma / 2-y & \beta \\ \gamma & \sigma / 2+y\end{array}\right|$, where $\sigma=\operatorname{tr} N$. The equalities $N(-1)=-1$ and $N(\omega)=\omega$ imply that $\beta=\gamma \omega$ and $y=(1-\omega) \gamma / 2$. Since

$$
\operatorname{det} N=\sigma^{2} / 4-\gamma^{2}(1+\omega)^{2} / 4
$$


we have

$$
\gamma^{2}=4\left(\sigma^{2} / 4-\operatorname{det} N\right) /(1+\omega)^{2} .
$$

Since $\sigma$ and det $N$ are real by the hypothesis $N \in \Omega, \gamma$ is real or purely imaginary. If $\gamma \in \mathbb{R}$, then $N$ is real, so suppose that $\gamma$ is purely imaginary. Let $S=\left|\begin{array}{cc}i / \sqrt{-\omega} & 1 \\ 1 & i \sqrt{-\omega}\end{array}\right|$. Then $S$ is an $L$-map and $S N S^{-1}=\left|\begin{array}{cc}\sigma / 2-z & z(1-\omega) / 2 \omega \\ z(1-\omega) / 2 & \sigma / 2+z\end{array}\right|$, where $z=i \gamma \sqrt{-\omega}$. Since this matrix is real, the proof is complete.

\section{Lemmas on real matrices in $\boldsymbol{\Omega}$.}

Lemma 5. Let $N=\left|\begin{array}{ll}w & x \\ y & z\end{array}\right|$ have real entries. Then $N \in \Lambda$ if and only if $N \in P$.

Proof. Suppose that $N \in \Lambda$. It was shown in $\$ 3$ that $\Lambda=\Lambda^{-1}$. Hence $N^{-1}(\infty) \in \operatorname{cl}\left(L^{-}\right)$, so that either $y, z \geq 0$ or $y, z \leq 0$. Further, for all $t>0$,

$$
\frac{w t+x}{y t+z}<0
$$

It follows that $N \in P$.

Conversely, suppose that $N \in P$. Then $N^{-1}(\infty) \notin L^{+}$, so for all $\tau \in L^{+}$,

$$
\operatorname{sgn} \operatorname{Re}(N(\tau))=\operatorname{sgn}\left\{w y|\tau|^{2}+x z+(x y+w z) \operatorname{Re} \tau\right\}<0 .
$$

It follows that $N \in \Lambda$. This completes the proof.

The next lemma characterizes those matrices $M \in \Omega$ which map $L_{M}$ onto a straight line. (Recall that $M$ always denotes a matrix $\left|\begin{array}{ll}a & b \\ c & d\end{array}\right| \in \Omega$ with $a, b \geq 0$, and $c, d \leq 0$.)

LEMMA 6. The following are equivalent:

(i) $\infty \in M\left(L_{M}\right)$;

(ii) $M=W_{b}$, or both $\operatorname{tr} M=0$ and det $M=1$;

(iii) $M\left(L_{M}\right)=L_{M}$.

Proof. We show that (i) $\Rightarrow$ (ii) $\Rightarrow$ (iii) (note that (iii) $\Rightarrow$ (i) is obvious).

Case 1: det $M=1$. Suppose that $\infty \in M\left(L_{M}\right)$. If $M(\infty)=\infty$, then $M=\left|\begin{array}{ll}a & b \\ 0 & d\end{array}\right|$, where $a>0$ and $d<0$. This contradicts the fact that det $M=1$. Hence $M(\infty) \neq \infty$, i.e., $c<0$. Then $-d / c \in L_{M}$, so $-d / c=\operatorname{Re} \omega_{M}$. By definition of $\omega_{M}$,

$$
-d / c=\operatorname{Re} \omega_{M}=\operatorname{Re}\left\{(2 c)^{-1}\left(a-d-\sqrt{(a+d)^{2}-4}\right)\right\} .
$$

It is readily seen that (1) holds if and only if $a+d=0$. This proves (ii).

To prove that (ii) $\Rightarrow$ (iii), suppose that $\operatorname{tr} M=0$. Then $c \neq 0$ and by the second equality 
in $(1)$,

$$
M(\infty)=a / c=\operatorname{Re} \omega_{M}=\operatorname{Re} \omega_{M}^{\prime} .
$$

Thus $M$ maps each of $\omega_{M}, \omega_{M}^{\prime}$ and $\infty$ into $L_{M}$, so $M\left(L_{M}\right)=L_{M}$.

Case 2: $\operatorname{det} M=-1$. In this case, $\omega_{M}, \omega_{M}^{\prime} \in \mathbb{R}^{*}$. Suppose that (i) holds. Assume that $c<0$. Then $-d / c \in L_{M}$, so $-d / c=\operatorname{Re} \omega_{M}=\omega_{M}$. Then

$$
-d / c=\omega_{M}=\left(a-d-\sqrt{\left.(a+d)^{2}+4\right)} / 2 c\right.
$$

which is impossible. Hence $c=0$, i.e., $M(\infty)=\infty$. By Lemma $3, M=W_{b}$. This proves (ii).

To prove that (ii) $\Rightarrow$ (iii), note that if $M=W_{b}$, then $M$ fixes the line $\{z: \operatorname{Re} z=-b / 2\}=$ $L_{M}$. This completes the proof.

Define II to be the set of (real) $M \in \Omega$ which satisfy (ii) of Lemma 6. Define $D_{M}$ as follows. If $M \in \Pi$, let $D_{M}=L_{M}^{-}$. If $M \in \Omega_{4}$, let $D_{M}$ be the interior of the circle $M^{2}\left(L_{M}\right)$. For all other $M \in \Omega$, let $D_{M}$ be the interior of the circle $M\left(L_{M}\right)$. If $M \in \Omega_{4}$, define $D_{M}^{\prime}$ to be the interior of the circle $M\left(L_{M}\right)$. (See Figures 1 through 5.) Note that since $M$ is real and conformal, the circles $M\left(L_{M}\right)$ and $M^{2}\left(L_{M}\right)$ are orthogonal to the real axis.

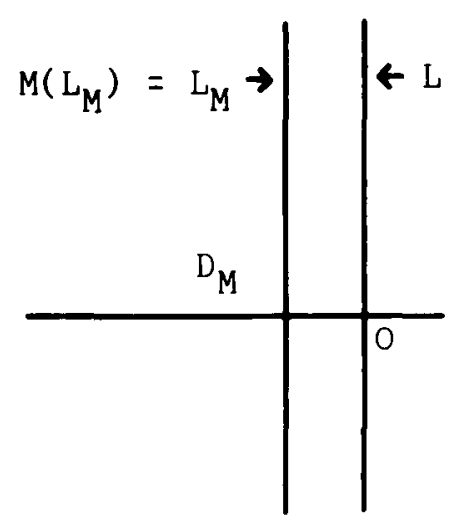

Figure 1. $M \in \Pi$

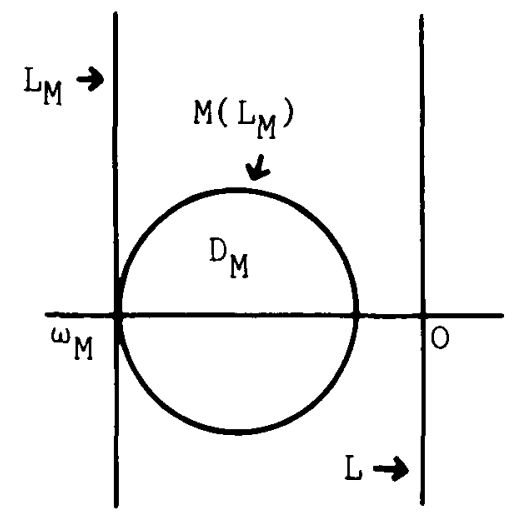

Figure 2. $M \in \Omega_{1}, M$ plussed

Lemmas 7 through 11 (below) show that if $M$ is plussed (recall that this means $c(a+d) \geq 0)$, then $M\left(L_{M}^{+}\right)=D_{M}$ when $M \notin \Omega_{4}$, and $M\left(L_{M}^{+}\right)=D_{M}^{\prime}$ and $M^{2}\left(L_{M}^{+}\right)=D_{M}$ when $M \in \Omega_{4}$. Lemmas 8 through 11 also verify that $D_{M}$ and $D_{M}^{\prime}$ are positioned as suggested by Figures 2 through 5 . We note that if $\omega_{M} \in L$, then $M \in \Omega_{3}$. For if $\omega_{M}=i r$, then $M=$ $\left|\begin{array}{cc}0 & -r \\ 1 / r & 0\end{array}\right|$ when $r>0$, and $M$ is as given in Lemma 3 when $r=0$.

LEMMA 7. If $M \in \Pi$, then $M\left(L_{M}^{+}\right)=D_{M}$.

Proof. First suppose that $M=W_{b}$. Then $M$ maps the points $-b / 2,-b / 2+i, \infty$ to $-b / 2$, $-b / 2-i, \infty$, respectively. Thus $M$ fixes $L_{M}$ but reverses its orientation. Therefore $M\left(L_{M}^{+}\right)=$ $L_{M}^{-}=D_{M}$. 


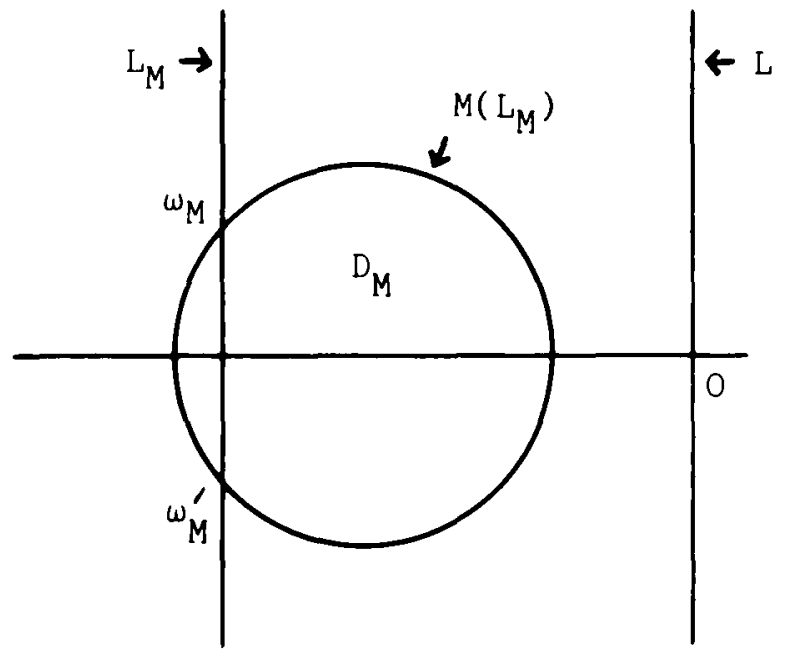

Figure 3. $M \in \Omega_{2}, M$ plussed

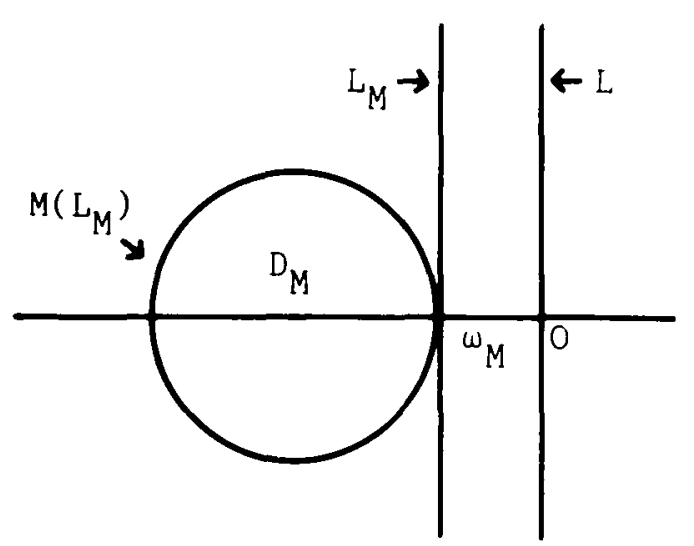

Figure 4. $M \in \Omega_{3} \backslash \Pi$

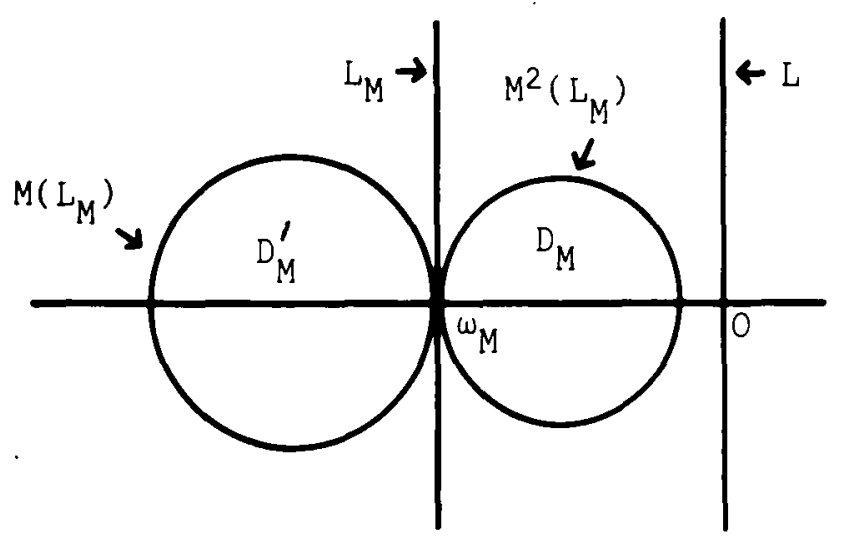

Figure 5. $M \in \Omega_{4}, M$ plussed 
Now suppose that $M=\left|\begin{array}{rr}a & b \\ c & -a\end{array}\right|$ with $\operatorname{det} M=1$. If $c=0$, then $\operatorname{det} M<0$; hence $c \neq 0$. Therefore, $M$ maps the points $(a-i) / c, a / c,(a+i) / c$ to $(a-i) / c, \infty,(a+i) / c$, respectively. Consequently, $M$ fixes $L_{M}$ but reverses its orientation, so $M\left(L_{M}^{+}\right)=L_{M}^{-}=D_{M}$. This completes the proof.

LeMma 8. If $M \in \Omega_{1}$ is plussed, then $D_{M} \subset L^{-} \cap L_{M}^{+}$and $M\left(L_{M}^{+}\right)=D_{M}$.

Proof. It suffices to show that $\omega_{M}<M(\infty) \leq 0$. For then, clearly $D_{M} \subset L^{-} \cap L_{M}^{+}$. Also, since the upper half-plane is invariant under $M$, the sequence $M\left(\omega_{M}\right), M\left(\omega_{M}+i\right), M(\infty)$ will determine a clockwise orientation on $M\left(L_{M}\right)$, so $M\left(L_{M}^{+}\right)=D_{M}$.

If $c=0$, then by Lemma $3, M=W_{\rho}$ for some $\rho$. Then det $M=-1$, which contradicts the fact that $M \in \Omega_{1}$. Hence $c<0$. As $M$ is plussed, $\sigma=a+d<0$. Hence $\sqrt{\sigma^{2}-4}<-\sigma$. Dividing by $-2 c$ and then adding $(a-d) / 2 c$, we have

$$
\omega_{M}=\left(a-d-\sqrt{\sigma^{2}-4}\right) / 2 c<a / c=M(\infty) .
$$

Finally, $a / c \leq 0$ because $M \in P$. This completes the proof.

Lemma 9. If $M \in \Omega_{2}$ is plussed, then $D_{M} \subset L^{-}$and $M\left(L_{M}^{+}\right)=D_{M}$.

Proof. It suffices to show that $\operatorname{Re} \omega_{M}<M(\infty) \leq 0$. As shown in the proof of Lemma 8 , $c<0, a / c \leq 0$, and $a+d<0$. Thus,

$$
\operatorname{Re} \omega_{M}=(a-d) / 2 c<a / c=M(\infty) \leq 0 .
$$

LeMma 10. If $M \in \Omega_{3} \backslash \Pi$, then $D_{M} \subset L_{M}^{-}$and $M\left(L_{M}^{+}\right)=D_{M}$.

Proof. It suffices to show that $M(\infty)<\omega_{M}$. For then, clearly $D_{M} \subset L_{M}^{-}$. Also, since $M$ maps the upper half-plane to the lower half-plane, the sequence $M\left(\omega_{M}\right), M\left(\omega_{M}+i\right), M(\infty)$ will determine a clockwise orientation on $M\left(L_{M}\right)$, so $M\left(L_{M}^{+}\right)=D_{M}$.

If $c=0$, then $M=W_{\rho}$ for some $\rho$, which contradicts the fact that $M \notin \Pi$. Hence $c<0$ and

$$
M(\infty)=a / c<(a-1) / c=\omega_{M}
$$

This completes the proof.

LeMma 11. If $M \in \Omega_{4}$ is plussed, then $D_{M}^{\prime} \subset L_{M}^{-} D_{M} \subset L^{-} \cap L_{M}^{+}, M\left(L_{M}^{+}\right)=D_{M}^{\prime}$, and $M\left(D_{M}^{\prime}\right)=D_{M}$.

Proof. As in the proof of Lemma 10, it suffices to show that

$$
M(\infty)<\omega_{M}<M^{2}(\infty) \leq 0 .
$$

Write $M^{2}=\left|\begin{array}{ll}a_{2} & b_{2} \\ c_{2} & d_{2}\end{array}\right|$. Since $M$ is plussed, $c_{2}=c(a+d) \geq 0$. Also, $a_{2}+d_{2}=(a+d)^{2}+2 \geq 2$. This proves that $M^{2}$ is plussed and that $M^{2} \in \Omega_{1}$. Thus, the inequality $\omega_{M}<M^{2}(\infty) \leq 0$ follows exactly as in the proof of Lemma 8.

If $c=0$, then by Lemma $3, M=W_{\rho}$, which contradicts the fact that $M \in \Omega_{4}$. Hence 
$c<0$. Clearly,

$$
-(d+a)<\sqrt{(a+d)^{2}+4} .
$$

Dividing by $-2 c$ and then adding $(a-d) / 2 c$, we have

$$
M(\infty)=a / c<\left(a-d-\sqrt{(a+d)^{2}+4}\right) / 2 c=\omega_{M} .
$$

This completes the proof.

LEMma 12. Define $\mathcal{O}_{M}=L_{M}^{-} \cup D_{M}$. If $M$ is plussed, then $M^{n}\left(\mathrm{ccl} \mathcal{O}_{M}\right) \subset O_{M}$ for all $n$ such that $M^{n} \neq I$.

Proof. Case 1: $M \in \Omega_{1}$. We have $M\left(L_{M}^{+}\right)=D_{M} \subset L_{M}^{+}$by Lemma 8 , and hence

$$
M^{n}\left(L_{M}^{+}\right) \subset D_{M} \subset \mathcal{O}_{M} \quad(n>0) .
$$

Also, $M^{-1}\left(\operatorname{ccl} D_{M}\right)=L_{M}^{-} \subset \operatorname{ccl} D_{M}$, and hence

$$
M^{-n}\left(\operatorname{ccl} D_{M}\right) \subset L_{M}^{-} \subset O_{M} \quad(n>0) .
$$

Since $\operatorname{ccl} O_{M}=L_{M}^{+} \cap \operatorname{ccl} D_{M}$, the desired result follows from (2) and (3).

Case 2: $M \in \Omega_{2}$. Let $q$ be the order of $M$. Fix $\tau \in \operatorname{ccl} O_{M}$. Let $K_{\tau}=$ $\left\{z \in \mathbb{C}^{*}:\left|t_{1}(z)\right|=\left|t_{1}(\tau)\right|\right\}$ where $t_{1}=\left|\begin{array}{ll}1 & -\omega_{M} \\ 1 & -\omega_{M}^{\prime}\end{array}\right|$. If $\tau \in \mathbb{R}$, then $K_{\tau}=\mathbb{R}^{*}$. If $\tau \notin \mathbb{R}$, then $K_{r}$ is a circle through $\tau$ such that $M\left(K_{\tau}\right)=K_{\tau}$. (This can be shown using the formula $t(W(\tau))=$ $\rho^{-2} t(\tau)$ which occurs in $\left[1\right.$, p. 112].) If $\operatorname{Im} \tau>0$, then $K_{\tau} \subset\{z: \operatorname{Im} z>0\}$ and $\omega_{M}$ is inside $K_{r}$; if $\operatorname{Im} \tau<0$, then $K_{\tau} \subset\{z: \operatorname{Im} z<0\}$ and $\omega_{M}^{\prime}$ is inside $K_{\tau}$. Thus $K_{r} \cap O_{M}$ is an arc or a ray. (See Figure 6.) By Lemma $9, M\left(L_{M}^{+}\right)=D_{M}$ and hence also $M^{-1}\left(\operatorname{ccl} D_{M}\right)=L_{M}^{-}$. Consequently, $M(\tau) \in O_{M}$ and $M^{-1}(\tau) \in O_{M}$. We claim that the points $\tau, M(\tau), \ldots, M^{q-1}(\tau)$ occur in that cyclic order on $K_{r}$. To see this, choose a complex linear fractional transformation $Y$ such that $Y(\infty)$ and $Y(0)$ are the fixed points of $M$. Then $X=Y^{-1} M Y=$ $\left|\begin{array}{cc}u & 0 \\ 0 & 1 / u\end{array}\right|$ for some complex $u \neq 0$. We have $\alpha=X^{q}(\alpha)=u^{2 q} \alpha$ for all $\alpha$, so $u^{2 q}=1$. Since

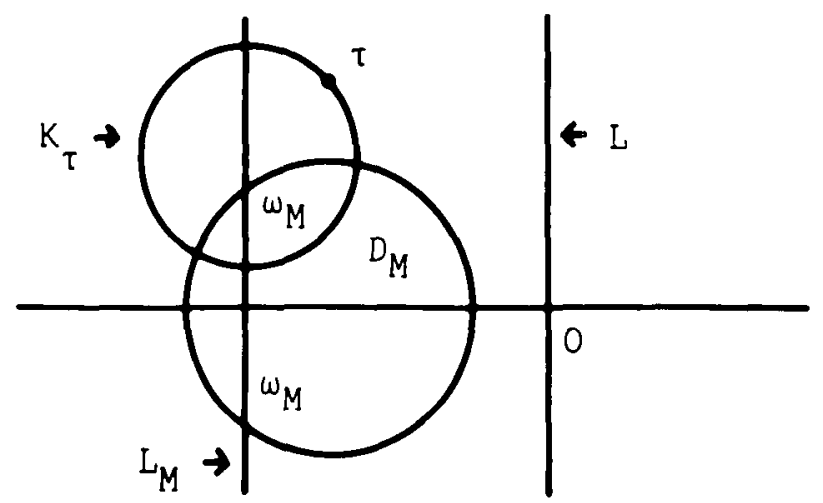

Figure 6 
$M \in \Omega_{2}, u^{2}=\exp ( \pm 2 \pi i / q)$. Let $\alpha=Y^{-1}(\tau)$. The points $\alpha, X(\alpha), X^{2}(\alpha), \ldots, X^{q-1}(\alpha)$ occur in that cyclic order on $Y^{-1} K_{r}$. This proves the claim. It follows that the points $M(\tau), \ldots, M^{q-1}(\tau)$ all lie on $K_{\tau} \cap O_{M}$. Thus $M^{n}(\tau) \in O_{M}$ for all $n$ such that $M^{n} \neq I$.

Case 3: $M \in \Omega_{3}$. By Lemmas 7 and 10,

$$
M\left(\operatorname{ccl} O_{M}\right)=M\left(L_{M}^{+}\right)=D_{M} \subset L_{M}^{-}=O_{M} .
$$

Since $M$ is an involution, this is the desired result.

Case 4: $M \in \Omega_{4}$. By Lemma 11, $M^{2}\left(L_{M}^{+}\right)=D_{M} \subset L_{M}^{+}$, and hence

$$
M^{2 n}\left(L_{M}^{+}\right) \subset D_{M} \subset O_{M} \quad(n>0) .
$$

Also, $M^{-2}\left(\operatorname{ccl} D_{M}\right)=L_{M}^{-} \subset \operatorname{ccl} D_{M}$, and hence

$$
M^{-2 n}\left(\operatorname{ccl} D_{M}\right) \subset L_{M}^{-} \subset \mathcal{O}_{M} \quad(n>0) .
$$

For all $n>0$, we have, by (5) and Lemma 11 ,

$$
M^{-2 n+1}\left(L_{M}^{+}\right)=M^{-2 n}\left(D_{M}^{\prime}\right) \subset M^{-2 n}\left(\operatorname{ccl} D_{M}\right) \subset \mathcal{O}_{M} .
$$

For all $n>0$, we have, by (4) and Lemma 11,

$$
M^{2 n-1}\left(L_{M}^{+}\right) \subset M^{-1}\left(D_{M}\right)=D_{M}^{\prime} \subset L_{M}^{-} \subset O_{M} \text {. }
$$

The desired result now follows from (4), (5), (6) and (7). This completes the proof.

\section{Proofs of Theorems 1 and 2.}

Proof of Theorem 1. By Theorem 4, there exist $L$-maps $Q_{1}$ and $Q_{2}$ such that $X=Q_{1}^{-1} A Q_{1}$ and $Y=Q_{2}^{-1} B Q_{2}$ are real. Since $A \in \Omega$ and $Q_{1}$ is an $L$-map, $X \in \Omega$. Similarly, $Y \in \Omega$. We may assume without loss of generality that $X$ is plussed, otherwise replace $A$ by $A^{-1}$. Similarly, assume that $Y$ is plussed. Let $Z=Y^{W}$ (recall that $\left.W=\left|\begin{array}{rr}1 & 0 \\ 0 & -1\end{array}\right|\right)$. Note that $Z=Q_{3}^{-1} C Q_{3}$, where $Q_{3}=Q_{2}^{W}$. Define $O_{X}$ and $O_{Y}$ as in Lemma 12. Note that $O_{X} \subset L^{-}$and $W\left(O_{Y}\right) \subset L^{+}$(see Figures 1 through 5). Since $Q_{1}$ and $Q_{3}$ are $L$-maps, $O_{1}=Q_{1}\left(O_{X}\right) \subset L^{-}$and $O_{3}=Q_{3}\left(W\left(O_{Y}\right)\right) \subset L^{+}$. Define $F=\operatorname{ccl}\left(O_{1} \cup O_{3}\right)$. Since, by hypothesis, at least one of $A$ and $C$ fixes no point of $L$, we have $F \neq \varnothing$. To see this, suppose, for example, that $A$ fixes no point of $L$. Then $X$ fixes no point of $L$, so $L^{-} \cap \operatorname{ccl}\left(\mathcal{O}_{X}\right) \neq \varnothing$ (see Figures 1 through 5). Thus $L^{-} \cap \operatorname{ccl}\left(O_{1}\right) \neq \varnothing$, so $F \neq \varnothing$.

To show that $\langle A, C\rangle$ is the discrete free product $\langle A\rangle *\langle C\rangle$, we will show that for every nontrivial reduced word $U$ in $\langle A, C\rangle, U(F) \cap F=\varnothing$. To show this, it suffices to prove that for all integers $n$ such that $A^{n} \neq I$,

$$
A^{n}\left(F \cup \mathcal{O}_{3}\right) \subset \mathcal{O}_{1},
$$

and that for all integers $n$ such that $C^{n} \neq I$,

$$
C^{n}\left(F \cup \mathcal{O}_{1}\right) \subset \mathcal{O}_{3} \text {. }
$$

We give an example to illustrate why it suffices to prove (8) and (9). Let $\tau \in F$ and let $U$ 
be the reduced word $A^{5} C^{-3} A^{4}$. By (8), $\tau_{1}=A^{4} \tau \in \mathcal{O}_{1}$. By (9), $\tau_{2}=C^{-3} \tau_{1} \in \mathcal{O}_{3}$. By (8), $U(\tau)=A^{5} \tau_{2} \in \mathcal{O}_{1}$, so $U(\tau) \notin F$. Since

Putting $M=X$ in Lemma 12 , we have $X^{n}\left(\operatorname{ccl} \mathscr{O}_{X}\right) \subset \mathscr{O}_{X}$ for all $n$ such that $X^{n} \neq I$.

$$
Q_{1}\left(\operatorname{ccl} \mathscr{O}_{X}\right)=\operatorname{ccl}\left(Q_{1}\left(\mathscr{O}_{X}\right)\right)=\operatorname{ccl}\left(\mathscr{O}_{1}\right),
$$

it follows that $A^{n}\left(\operatorname{ccl} \mathscr{O}_{1}\right) \subset \mathcal{O}_{1}$, for all $n$ such that $A^{n} \neq I$. This yields (8). Putting $M=Y$ in Lemma 12 , we have $W Z^{n} W\left(\operatorname{ccl} O_{Y}\right) \subset O_{Y}$ for all $n$ such that $Z^{n} \neq I$. Hence $C^{n}\left(\operatorname{ccl} O_{3}\right) \subset O_{3}$ for all $n$ such that $C^{n} \neq I$. This yields (9) and completes the proof.

Before proving Theorem 2, we prove the following lemma.

Lemma 13. Let $A$ and $C$ be linear fractional transformations of order 2 such that $A \neq C$ and $A C$ is not elliptic. Then $\langle A, C\rangle$ is the discrete free product $\langle A\rangle *\langle C\rangle$.

Proof. The reduced words in $\langle A, C\rangle$ are the words alternating in the symbols $A$ and $C$, e.g., ACACACA. Since $A \neq C, A C \neq I$. Since $A C$ is not elliptic, $A C$ has infinite order. Hence $\langle A, C\rangle=\langle A\rangle *\langle C\rangle$. Moreover, by [3, Theorem 1E, p. 87], $\langle A C\rangle$ is discrete. Since $[\langle A, C\rangle:\langle A C\rangle]=2$, it follows that $\langle A, C\rangle$ is discrete. This completes the proof.

Proof of Theorem 2. If $\{A, C\} \subset\left\{W, T_{u}\right\}$ for some $u$, clearly $\langle A, C\rangle$ is not the free product of $\langle A\rangle$ and $\langle C\rangle$. Conversely, assume that for every $u,\{A, C\} \not \subset\left\{W, T_{u}\right\}$. It may be assumed that both $A$ and $C$ have a fixed point on $L$, otherwise $\langle A, C\rangle$ is the discrete free product $\langle A\rangle *\langle C\rangle$ by Theorem 1 . As $W(L)=L, B$ also has a fixed point on $L$. Let $M \in\{A, B\}$ (where we write $M=\left|\begin{array}{ll}a & b \\ c & d\end{array}\right|$ with $a, b \geq 0$ and $c, d \leq 0$ ). If $M$ fixes 0 or $\infty$, then $M=W_{b}$ (with $b \geq 0$ ) or $M=W_{c}^{t}$ (with $c \leq 0$ ) by Lemma 3. If $M$ fixes a point $u i$ $(0<u<\infty)$, then an easy calculation shows that $M=\left|\begin{array}{cc}a & -c u^{2} \\ c & a\end{array}\right|$. But since the diagonal elements of $M$ cannot both be positive or negative, $a=0$. Hence $M=T_{u}$. Since for every $u$, $\{A, C\} \not \subset\left\{W, T_{u}\right\},\langle A, C\rangle$ is one of the following groups or their transposes:

$$
\begin{array}{ll}
G_{1}=\left\langle W_{r}, W_{s}\right\rangle & (r \leq 0, s>0), \\
G_{2}=\left\langle W_{r}^{t}, W_{s}\right\rangle & (r>0, s>0), \\
G_{3}=\left\langle W_{s}, T_{u}\right\rangle & (s \neq 0),
\end{array}
$$

or

$$
G_{4}=\left\langle T_{u}, T_{v}\right\rangle \quad(u>v>0) .
$$

Let $U_{i}$ and $V_{i}$ be the first and second given generator of $G_{i}$, respectively ( $i=$ $1,2,3,4)$. It remains to show that each $G_{i}$ is the discrete free product $\left\langle U_{i}\right\rangle *\left\langle V_{i}\right\rangle$. It is easily checked that $U_{i}$ and $V_{i}$ are involutions and that $U_{i} V_{i}$ is not elliptic $(i=1,2,3,4)$. (Note, for example, that $U_{3} V_{3}$ is loxodromic.) Hence the result follows from Lemma 13 and the proof is complete. 
7. Transformations with fixed points on $L$. We discuss the structure of $\langle A, C\rangle$ where $A \in \Omega, C \in \Omega^{\mathrm{W}}$, and both $A$ and $C$ have a fixed point on $L$. The case where these fixed points are equal is treated in Theorem 14; the remaining case is treated in Theorem 15.

Theorem 14. Let $A \in \Omega$ and $C \in \Omega^{w}$. Suppose that $A$ and $C$ both fix $\omega \in L$. Then $\langle A, C\rangle$ is the discrete free product $\langle A\rangle *\langle C\rangle$ unless $A=C$.

Proof. If $\omega \neq \infty$, conjugate $A$ and $C$ by the $L$-map $\left|\begin{array}{rr}0 & 1 \\ 1 & -\omega\end{array}\right|$. Thus we may assume without loss of generality that $\omega=\infty$. By Lemma $3, A=W_{\rho}$ and $C=W_{v}^{w}=W_{-\nu}$ for some pair $\rho, \nu$. Suppose that $A \neq C$. Then $A C=\left|\begin{array}{ll}1 & u \\ 0 & 1\end{array}\right|$ for some $u \neq 0$. The result thus follows
from Lemma 13 .

Theorem 15. Let $A \in \Omega$ and $C \in \Omega^{w}$. Suppose that $A$ fixes $\omega_{A} \in L$ and $C$ fixes $\omega_{C} \in L$, where $\omega_{A} \neq \omega_{C}$. Then there exists an $L$-map $S$ such that $A^{S}=W_{\rho}$ and $C^{S}=W_{v}^{t}$ for some pair $\rho, \nu$. Also $\langle A, C\rangle$ is the discrete free product $\langle A\rangle *\langle C\rangle$ if and only if $A \neq C$ and $A C$ is not elliptic.

Proof. We begin by proving the first assertion. If $\omega_{A} \neq \infty$, conjugate $A$ and $C$ by the $L$-map $\left|\begin{array}{cc}0 & 1 \\ 1 & -\omega_{A}\end{array}\right|$. Thus it may be assumed without loss of generality that $\omega_{A}=\infty$. Let $S$ be the L-map $\left|\begin{array}{cc}1 & -\omega_{C} \\ 0 & 1\end{array}\right|$ (note that $\omega_{C} \neq \omega_{A}=\infty$ ). Then $A^{s}(\infty)=\infty$ and $C^{S}(0)=0$. Since $\left(C^{s}\right)^{W} \in \Omega$ and $\left(C^{s}\right)^{W}(0)=0$, it follows from Lemma 3 that $A^{s}=W_{\rho}$ for some $\rho$ and $C^{\mathrm{WS}}=W_{-\nu}^{t}$ for some $\nu$. Hence $C^{S}=W_{\nu}^{t}$.

To prove the second assertion, assume that $A C$ is elliptic. If $A C$ has finite order, then $\langle A, C\rangle$ is not the free product $\langle A\rangle *\langle C\rangle$. If $A C$ has infinite order, then $\langle A C\rangle$, and hence $\langle A, C\rangle$, is not discrete. Conversely, assume that $A \neq C$ and that $A C$ is not elliptic. By the first assertion of this theorem, $A$ and $C$ are involutions. Thus $\langle A, C\rangle$ is the discrete free product $\langle A\rangle *\langle C\rangle$ by Lemma 13 .

\section{REFERENCES}

1. R. J. Evans, A fundamental region for Hecke's modular group, J. Number Theory 5 (1973), 108-115.

2. R. J. Evans, Free products of two real cyclic matrix groups, Glasgow Math. J. 15 (1974), 121-128.

3. J. Lehner, Discontinuous groups and automorphic functions, Math. Surveys of the Amer. Math. Soc. 8 (Providence, R.I., 1964).

4. M. Newman, Integral matrices (Academic Press, 1972).

5. N. Purzitsky, Two-generator discrete free products, Math. Z. 126 (1972), 209-223.

6. N. Purzitsky, All two-generator Fuchsian groups, Math. Z. 147 (1976), 87-92.

Mathematics Department

University of California, San Diego

La Jolla, California 92093 